Radiologe 2009 · 49:675

DOI 10.1007/s00117-009-1909-9

Online publiziert: 20. August 2009

(c) Springer Medizin Verlag 2009

\author{
H.-U. Kauczor ${ }^{1} \cdot$ H. Imhof ${ }^{2}$ \\ ${ }^{1}$ Abteilung Diagnostische und Interventionelle Radiologie, \\ Universitätsklinikum Heidelberg \\ ${ }^{2}$ Univ.-Klinik für Radiodiagnostik, AKH, Medizinische Universität Wien
}

\title{
Regionale Messung der Lungenfunktion
}

\section{Die Rolle radiologischer Verfahren}

Funktionelle Verfahren in der CT und MRT sind schon lange nicht mehr nur auf das Gehirn beschränkt. Mehr und mehr Organsysteme und onkologische Erkrankungen lassen sich mit modernen multidimensionalen Methoden funktionell und quantitativ untersuchen. Lunge und Gasaustausch stellen hierbei über die rein strukturelle Darstellung hinaus [1] große Herausforderungen an den Radiologen. Dieses Themenheft hat sich die Aufgabe gestellt, aktuelle Entwicklungen und Anwendungsgebiete zusammenfassend darzustellen und ergänzt damit hervorragend bereits vorliegende Themenhefte mit Originalarbeiten [3].

Als allgemein akzeptiertes und verfügbares Verfahren stellt die traditionelle Lungenfunktionsprüfung mit Spirometrie und Bodyplethysmographie die Messlatte für die regionalen radiologischen Verfahren dar (Beitrag Sorichter). Dichtebasierte Auswertungen in der CT werden schon seit geraumer Zeit genutzt, um den „Ventilationszustand" der Lunge zu beschreiben [2]. Heute sind Protokolle und Auswertealgorithmen etabliert, die bei beatmeten Patienten mit akutem Lungenversagen auch routinemäßig zum Einsatz kommen (Beitrag Reske u. Seiwerts).

Moderne MDCT-Scanner erlauben auch dynamische Untersuchungen der gesamten Lunge während fortgesetzter normaler oder forcierter Atmung. Bei Verwendung eines entsprechenden Signals lassen sich prospektiv oder retrospektiv atemgetriggerte Aufnahmen berechnen. Dieser Technik kommt in der modernen Strahlentherapieplanung erhebliche Bedeutung $\mathrm{zu}$, wobei auch die er- höhte Strahlenexposition gerechtfertigt ist (Beitrag Dinkel et al.). Gleichzeitig gewinnt die MRT zunehmend Bedeutung zur funktionellen Untersuchung der Lunge und zeigt hervorragende Ergebnisse bei der Darstellung der Atembewegung pulmonaler Tumoren (Beitrag Biederer et al.). Gleichzeitig kann die MRT gezielt die Atemmechanik und regionale Lungenvolumina analysieren und eine sinnvolle Ergänzung der konventionellen Lungenfunktionsprüfung sein (Beitrag Tetzlaff u. Eichinger).

Für die direkte Abbildung der Ventilation stehen verschiedene aufwändige Techniken zur Verfügung. Eine detaillierte Analyse gelingt insbesondere nach Inhalation eines hyperpolarisierten Edelgases, wie ${ }^{3}$ Helium. Hiermit können die Ventilation statisch und dynamisch, die alveoläre Mikrostruktur und der regionale Sauerstoffpartialdruck gemessen werden (Beitrag Gast u. Wolf). Als einfacheres Verfahren lässt sich er Gasaustausch mit der sauerstoffverstärkten ${ }^{1} \mathrm{H}$-MRT besonders gut beurteilen (Beitrag Beer et al.). Die zweite wichtige Komponente zur Beurteilung der funktionellen Leistungsfähigkeit der Lunge ist die Perfusion. Sie lässt sich mittels einer kontrastmittelverstärkten multiphasischen MRT leicht beurteilen und quantitativ erfassen (Beitrag Attenberger et al.). Die Untersuchung der Perfusion gehört mittlerweile zum Basisprotokoll einer MRT der Lunge in der klinischen Routine.

Insgesamt zeigt dieses Themenheft die Machbarkeit, eine enorme Leistungsstärke und zukünftiges Potenzial funktioneller radiologischer Verfahren zur regio- nalen und quantitativen Erfassung einzelner Facetten der Lungenfunktion.

Zweifellos ist es allen Autoren sehr gut gelungen, die enormen Fortschritte der „bildgebenden“ Lungenfunktion dazustellen, wofür wir ihnen herzlichst danken. Wir sind überzeugt, dass diese Ausgabe eine entsprechende Resonanz bei unseren Lesern findet.

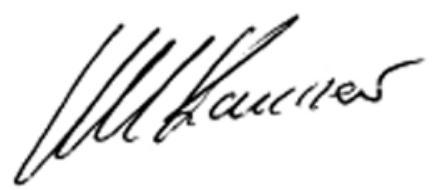

Prof. Dr. Hans-Ulrich Kauczor

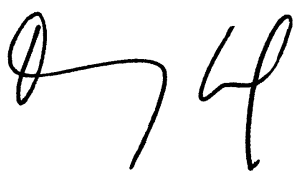

Prof. Dr. Herwig Imhof

\section{Literatur}

1. Reuter M, Biederer J (2009) Mustererkennung im hochauflösenden Computertomogramm (HRCT) der Lunge. Radiologe 49:159-172

2. Grosse C, Bankier A (2007) Bildgebung des Lungenemphysems. Radiologe 47:401-406

3. Goo JM, Kauczor HU, van Beek EJ (2008) Innovation in chest radiology. Invest Radiol 43:339-342 